\title{
Labyrinthe
}

$40 \mid 2013$

Comme les abeilles

\section{La métaphore économique de la pollinisation}

\section{Ariel Kyrou}

\section{OpenEdition}

\section{Journals}

Édition électronique

URL : http://journals.openedition.org/labyrinthe/4306

DOI : $10.4000 /$ labyrinthe.4306

ISSN : 1950-6031

Éditeur

Hermann

Édition imprimée

Date de publication : 1 mars 2013

Pagination : 41-42

ISBN : 9782705688400

Référence électronique

Ariel Kyrou, «La métaphore économique de la pollinisation », Labyrinthe [En ligne], 40 | 2013, mis en ligne le 01 mars 2015, consulté le 30 avril 2019. URL : http://journals.openedition.org/labyrinthe/4306 ; DOI : 10.4000/labyrinthe.4306

Propriété intellectuelle 


\title{
La métaphore économique de la pollinisation
}

\author{
Ariel KYrou
}

Revenons sur cette tendance à la métaphore entre société humaine et société des abeilles : il est clair que la métaphore que nous utilisons, Yann Moulier Boutang et moi, sur Google, est là juste pour faire réfléchir. Même chose quand je compare Google à « Dieu spinozien ». Les scientifiques purs et durs sont récalcitrants face à ce type d'images qui placent au même niveau des réalité sans rapport les unes avec les autres. On voit bien qu'il y a dans le monde internet une tendance qui va avec une vision des insectes moins négative que celle qu'on pouvait avoir à une certaine époque. On pourrait donner d'autres exemples : les logiciels libres, les Creative Commons, les œuvres en Copyleft, etc. mais aussi d'une certaine façon le sampling : ce sont toutes ces logiques d'enrichissement, de pollinisation des connaissances. On pourrait reprendre aussi les termes du sociologue Dominique Cardon, qui a beaucoup travaillé sur Wikipédia. Il explique qu'il voit dans Wikipédia et, globalement, sur internet un autre type de démocratie: ce n'est pas une démocratie représentative, avec le vote, mais une logique de décision concrète; c'est le processus même d'internet, se prendre en main pour créer soi-même sa propre encyclopédie. Quand on intervient dans Wikipédia, on peut modifier les textes - il faut simplement respecter des règles, sourcer ses textes, produire des textes neutres, etc. De même pour les abeilles : l'apiculteur ne les poursuit pas pour qu'elles rentrent dans la ruche. Wikipédia fonctionne sur cette même logique : une logique de la libre participation, une logique du faire qui donne à ceux qui font plus de pouvoir; mais c'est un pouvoir défini a posteriori.

Un autre point assez intéressant rejoint les théories du prix Nobel d'économie Elinor Ostrom, morte en juillet 2012: elle a travaillé sur les biens communs, les pêcheries. Elle a montré que les grandes pêcheries en Thaïlande fonctionnent sans qu'il y ait de chefs, avec une répartition, une distribution de la participation, qui est ouverte à tous, mais aussi une distribution de la sanction. C'est comparable chez Wikipédia: la sanction, chez Wikipédia, n'est pas dans la main de gens qui auraient le 


\section{Labyrinthe, $n^{\circ} 40$}

statut de policiers. C'est une différence de la société des abeilles avec la société des fourmis. Ces fonctions se jouent dans le temps de la vie des abeilles, dans une ruche, alors que, sur internet, elles se jouent dans le même temps : tout le monde est invité à avoir à la fois plusieurs fonctions (participer, critiquer, valider, etc.: on a pu parler de «Wikipompiers » pour désigner ceux qui devaient régler des conflits) : a priori, tout le monde peut agir et exister sur Wikipédia ou dans les pêcheries de Thaïlande; ce qui ne veut pas dire qu'a posteriori il n'y ait pas de différences. Ces différences se définissent a posteriori. Est-ce ainsi que cela se passe dans la société des abeilles ? Les meilleures productrices de miel ontelles a posteriori la préférence de la reine-mère et sont-elles in fine plus valorisées au sein de la ruche ? Honnêtement, je ne sais pas. Et je ne crois pas que cela soit grave de ne pas savoir... Car la métaphore ne pose pas une équivalence. Elle fonctionne comme un marchepied, ou sur un autre registre telle une fable.

Comparer notre nouveau monde numérique à un monde de fourmi ? Ce sera vécu comme un cauchemar, ou presque. Le comparer à un monde d'abeilles ? Alors même qu'elles sont en danger, les abeilles ont désormais une bonne image. La métaphore, très utile pour lire notre époque et ses évolutions, peut donc être vécue comme un rêve, ou presque. Mais au réveil, l'on aura beau mettre du miel sur sa tartine de pain et rouler mille fois des yeux devant son écran et le moteur de recherche Google, on n'en restera pas moins plus proche du cochon sur deux pattes ou du singe savant que des habitants volants des ruches des apiculteurs... 